Journal of Social Sciences 3 (4): 223-231, 2007

ISSN 1549-3652

(C) 2007 Science Publications

\title{
An Engineer's Mathematical Approach to Designing an Emergency Room Decontamination Area for Weapons of Mass Destruction Casualties
}

\author{
${ }^{1,2}$ Eldo E. Frezza, ${ }^{1}$ Erica Fletcher, ${ }^{1}$ Veronica Flores, ${ }^{1}$ Ellen Popolo and ${ }^{1}$ Fay Tal Placido \\ ${ }^{1,2}$ Department of Surgery, ${ }^{1}$ Engineering Institute, Texas Tech University, USA
}

\begin{abstract}
This study will focus on the potential requirements for an emergency response facility in case of weapons of mass destruction casualties proposing mathematical models. A complete emergency room layout will be designed taking the following analyses and requirements into consideration: quality function development (QFD) and product layout; average number of patients in the system, average time in the system, average number of patients in the waiting queue and average time waiting in the queue. Our analysis showed that, assuming 150 patients per day for 2 days with average service time of 14 minutes with two lines running. $\lambda=75$ arrivals $/$ day $=0.0521$ arrivals $/ \mathrm{min} . \mu$ $=2$ patients $/ 14 \mathrm{~min}=0.1429$ patients $/ \mathrm{min}$. Average number of patients in system (waiting and being served). Ls $=\lambda /(\mu-\lambda)=0.574$ patients. Average time in system (waiting and service time) $\mathrm{Ws}=1 /$ $(\mu-\lambda)=11.02 \mathrm{~min}$. Average number of patients waiting in queue. $\mathrm{Lq}=\lambda^{2} / \mu(\mu-\lambda)=0.209$ patients Average time waiting in queue, $\mathrm{Wq}=\lambda / \mu(\mu-\lambda)=4.016 \mathrm{~min}$. From our model, we conclude to set up an engineer's perfect ER response we need: Having a stable patient and unstable patient track will make it easy to respond to the emergency. Patients requiring the emergency track feature would be dropped off before the vehicle shower at contaminated stabilizing rooms. Time of service: waiting 14 minutes with an average of 2 patients waiting in line to be served. This is an initial study having a flow of 150 patients arriving over a long time period and it is an attempt to apply a mathematical model to a multidisciplinary approach to a clinical potential discussion.
\end{abstract}

Key words: emergency room layout; weapons of mass destruction; mass casualties

\section{INTRODUCTION}

Chemical warfare agents as a military issue are events and civilians could be exposed to these agents ${ }^{[1]}$. Weapons of mass destruction (WMD) are threats that all healthcare facilities must be prepared to handle ${ }^{[2]}$. Every healthcare facility is a vital part of the community response system and must be ready to respond. Private vehicles or busses might transport the majority of patients, with only a small percentage arriving by emergency medical service. Most will go to the hospital closest to the incident, even if it results in overcrowding. Others will go directly to their private physician's office or primary hospital even if these facilities are not part of the local disaster plan. Most of these victims will not be decontaminated before arrival. It is important to remember that in most cases of biological or chemical attacks, the victim will be unaware of what is causing their illness. Therefore, they will not be able to alert medical professionals. If the hospital allows any of these patients inside, the staff may be exposed to the toxin and the facility may require closure for decontamination ${ }^{[3-4]}$. The risk is universal. The healthcare facility must plan for communication that allows local government to transmit alerts regarding the emergency. Healthcare facilities must also communicate the status of the emergency needs to local officials during the emergency. In order to limit contamination, the facility must be prepared to establish a single entry control point and attempt to secure all other entrances. The ability to establish a patient decontamination team from on-duty staff with only a few minutes notice at any time of the day or night is vital ${ }^{[3]}$.

Many issues are considered as an absolute requirement for implementation of an NBC response: 1) policy, 2) plan and 3) emergency department response to surge capacity, including design of the emergency department, equipment required, personnel required, education and training, occupational health and safety issues and surveillance systems. This is the first attempt to have the medical profession approach the problem in a multidisciplinary fashion $^{[5]}$. 


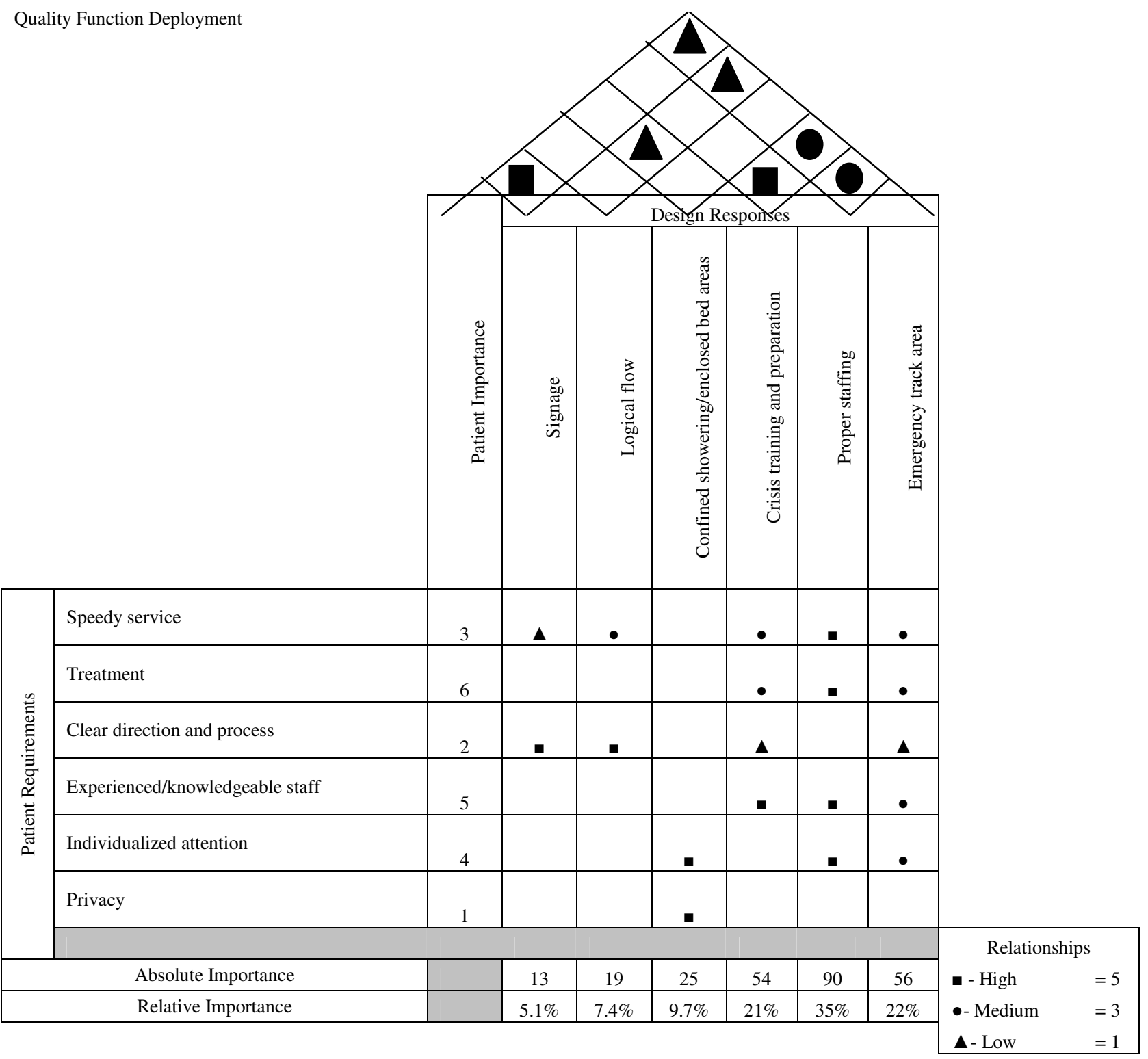

Fig. 1: Quality function development analysis

As stated in our previous study, "perhaps no form of disaster is more difficult to prepare for than one resulting from the international covert release of a biological pathogen or chemical toxin." As already reported, we need to build a response plan for the emergency room and the hospital emergency room ${ }^{[4]}$.

Aims: This study will focus on an engineer's mathematical approach to design an emergency response facility model only for contaminated vehicles and persons to handle such a disaster. Patients will be integrated into the existing emergency room facilities and follow standard protocol after decontamination processes. It is described how to add a decontamination area to a preexisting emergency room.

The study will include the engineering planning for an emergency facility layout addition, the flow of patients/staff/family and vehicles through various cleaning stations as well as space and personnel requirements. This is built with a mathematic approach to clinical problems with the flow of a perfect science applied to a clinical scenario, which is full of problems. 
Methods

Patient flow

Ambulance traffic: The regulation of movement of ambulances is very important. The emergency ambulance carries patients to one entrance, they stop at the drop-off point and then move away in one direction, assuring a smooth traffic flow. The emergency department should have the same system of one-way entrance to the emergency department where the decontamination should be continuous or treatment should be initiated $^{[3]}$.

The necessary process flow for the decontamination process will be: ambulance cleaning, shower patients and emergency medical technicians (EMTs) with clothes on, shower without contaminated clothes, changing station with fresh clothing, contaminated evaluation holding area for patients and inventory storage room for EMTs to restock supplies. This process will have two to three lanes with the ability to run simultaneously in order to accommodate multiple vehicles. For more critical patients, there will be a separate procedure implemented in which they will enter an "emergency track." Ambulances on this track will drop off the critically contaminated patients directly into the trauma room where they will be stabilized, assessed and decontaminated accordingly. This area will remain contaminated and staff within the area will be required to wear the appropriate personal protective equipment. The patients will then either be sent to an operating room or to a "step-down" area for further diagnosis and motoring. The ambulance itself will pass through one of the regular process flow lanes ${ }^{[6-9]}$

Adaptation to existing ER set-up: The number and width of the lanes will be determined using the dimensions of the existing hospital space. Shower stations will need proper dimensions to accommodate multiple people and will be within the existing driveway space as well. Layouts for equipment within trauma rooms, number of patient beds and proper walls will be designed to contain the contamination and provide for maximum patient capacity. Each section of the emergency room will be analyzed using process flow charts, product layout, quality function deployment diagram, relationship diagram and adjacency scoring in order to determine the proper space requirements and layout. A complete emergency room layout will be designed taking these analyses and requirements into consideration: quality function development (QFD), product layout, flow pattern, flow process chart, activity relationship diagram, relationship diagram and adjacency scoring.

In the beginning stages of planning for the ideal emergency room layout, a QFD analysis will be utilized
A. General Decontamination Area: Stable Patients 2 lanes $\rightarrow$ Product Layout



B. Emergency Track Contamination Area: Unstable Patients

1 lane $\rightarrow$ Product Layout



Fig. 2: A. Graphic decontamination area of stable patients, B. Graphic area of decontamination area of unstable patients

to determine the functional requirements and design parameters pertinent to the layout's needs. QFD is a concept that helps translate patient requirements into emergency service requirements. Initially, the patients' requirements were agreed upon and accordingly that knowledge was used to drive development and improvement of the process (Fig. 1). Before beginning any layout design, we had to identify the wants of the patients in an emergency chemical and biological attack situation to interpret the significant design responses of the hospital to ensure the optimal treatment of the patients. Under these circumstances, health services and the patients not only want decontamination treatment, but also speedy service, individualized attention, privacy, an experienced and knowledgeable staff and a clear process flow. To meet these patient requirements, the hospital should implement signage, a logical flow, confined showering and enclosed bed areas, crisis training and preparation, proper staffing and an emergency track area. The relationship between patient requirements and the design responses is shown above in the QFD analysis. Proper staffing, an emergency track area and crisis training and preparation prove to be the most vital in meeting the patients' needs.

Product layout:_It will be divided into two areas: general area for stable patients and emergency track for 
Table 1: Patient Flow Process Chart: For Stable Patient Flow

\begin{tabular}{|c|c|c|c|c|}
\hline 20 & Activity & Distance & Quantity & Notes \\
\hline $\begin{array}{l}\text { 1. Contaminated public enters emergency } \\
\text { response area }\end{array}$ & & & $\begin{array}{l}2 \text { ambulances } \\
\text { (maximum) }\end{array}$ & \\
\hline 2. Ambulance Shower & $D \Rightarrow$ & & 1 large shower & $\begin{array}{l}\text { Patient waits while ambulance } \\
\text { is being cleaned }\end{array}$ \\
\hline 3. Clothed patient shower & $\mathrm{O}$ & 130 & 5 individual showers & \\
\hline 4. Naked patient shower & $\mathrm{O}$ & 130 & 5 individual showers & $2 \mathrm{x}$ the capacity \\
\hline 5. Dressing area & $\mathrm{O}$ & 130 & 5 individual areas & \\
\hline 6. Emergency room & $\mathrm{D}$ & & & Nurse assessment \\
\hline \multicolumn{5}{|l|}{ Patient Flow Process Chart: For Unstable Patient Flow } \\
\hline Process & Activity & Distance (in) & Quantity & Notes \\
\hline $\begin{array}{l}\text { 1. Contaminated public enters emergency } \\
\text { response area } \\
\text { 2. Patients sent to emergency trauma beds }\end{array}$ & & 498 & $\begin{array}{l}2 \text { ambulances } \\
\text { (maximum) }\end{array}$ & \\
\hline 3. Patient treated at bed & $D \Rightarrow$ & & & \\
\hline 4. Clothed shower & $\mathrm{O}$ & & & \\
\hline 5. Naked shower & $\mathrm{O}$ & 114 & & \\
\hline 6. Dressing area & $O \Rightarrow$ & 114 & & \\
\hline 7. Step down area & $\mathrm{D}$ & 370 & & \\
\hline 8. Patient transferred to OR, ER, etc. & $\mathrm{D}$ & & & \\
\hline
\end{tabular}

unstable patients as reported in Fig. 2. The product layout reported in Fig. 2 details the flow of patients through the respective decontamination areas. These layouts minimize the distances a patient must travel, ultimately reducing confusion in time of a crisis. These layouts also allow for a smooth and logical flow as they correspond to the sequence of treatment procedures. These layouts most efficiently lessen the space occupied.

Flow process: To activate a stable area, we need to consider activity, distance in inches (in) and the quantity needed and create a flow process chart. The charts are reported in Table 1. Distance in inches and quantity of service showed the potential assessment of our project.

\section{Which factors affect flow pattern?}

Specific factors: There are many factors that can affect the flow pattern. It is advisable to consider every aspect of a design before implementing a design to ensure all issues are worked out beforehand.

There are two important factors that can affect the flow pattern of a facility: the types of flow patterns and the type of layout that is being implemented. There are five basic flow patterns including Straight, L Flow, U
Flow, Circular and S Flow. Each pattern has advantages and disadvantages. In most hospitals, a U Flow pattern is being implemented for the vehicles. Advantages to using the $U$ Flow are that it saves space and it is conducive to good communication. In some hospitals, a $\mathrm{U}$ Flow pattern is beneficial because incoming patients are able to see the results that people are being cared for and progress is being made. Also at each hospital considered, a product layout is being utilized, some of the advantages of using a product layout, as reported in Fig. 3, are:

* Logical flow lines because the layout corresponds to the sequence of operations.

* The total production time per unit is short.

* Material Handling is reduced because machinery is located to minimize distances.

* Work in transit and temporary storage occupies less space.

* Small in process inventories result, because the work from one process is fed directly into the next.

However, the most important disadvantage to consider is that if there is a breakdown somewhere in the line; it can lead to the complete stoppage of the line. As far as the patient flow, an L Flow pattern should be implemented. Here, the contaminated patients enter on one side of the decontamination area and exit on the 


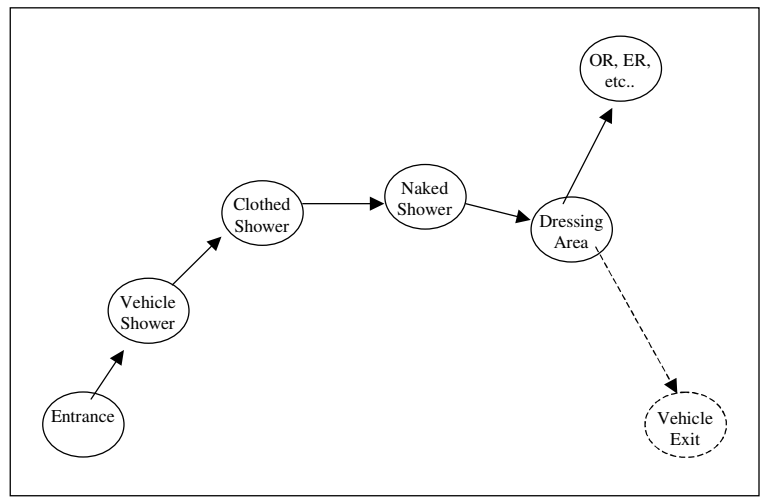

Fig. 3: Layout flow of vehicle and ER area

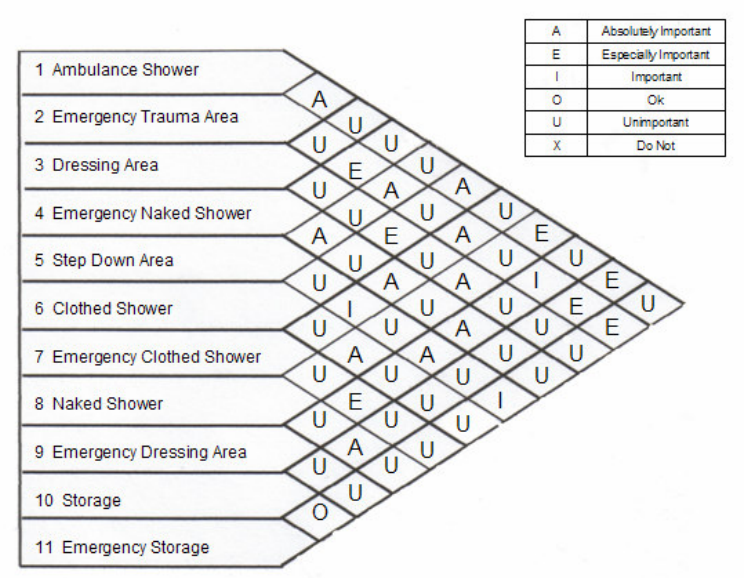

Fig. 4: Activity relationship diagram

opposite side as they are assessed to either the emergency room, operating room, etc.

\section{What problems does the facility have that can undermine the design? \\ Existing Factors}

Existing buildings: The five main existing factors in the facility that need to be considered are the following: It is important to consider existing buildings and structures. Planning new designs can be complicated because unlike machinery and workstations buildings cannot be moved. Also, when redesigning a plan it is much more cost effective to make use of existing materials, buildings, machines, workstations and other structures.

Necessary flow between work areas: In considering ideas for a new facility plan it is necessary to determine specific requirements for the order of operations. In the situation of redesigning an existing hospital emergency response area, it is difficult but important since vehicles and incoming patients must be decontaminated in a particular order as mentioned above in the problem statement.

Sequence of operations on each part: The sequence of operations on each part goes along with the necessary flow between work areas, because the necessary flow is a determining factor for the sequence of operations. However, in other facility plans a set sequence may not be required, so there would be more room to consider alternatives.

Materials storage: Material storage can play a large part in a new facility plan. The storage needed would be emergency trauma area storage and storage area for ambulances; both for restocking purposes. In a manufacturing or distribution center plan this would be a major factor impacting the flow pattern.

Desired Flexibility: Flexibility in a plan can help ensure that a design will be able to adjust to small everyday instances. In any hospital, there will be capabilities for minor flexibilities such as cars or ambulance decontamination or the number of male/female showers. Overall, some flexibility is desirable because small things can change and adaptation is required. Although, planning for a lot of flexibility is unwise because making changes after a plan is put into use can be very costly. It is advised to do a thorough study and observations examining every aspect of a facility and its particular needs before making any drastic changes.

An activity relationship diagram, as shown in Fig. 4 , is important to allow us to show the desired closeness of stations within the decontamination treatment area. Moreover, it gives the initial assessment before getting into the relationship diagram which will address which one is an important pathway. Only after the activity and the relationship diagram can we originate our decontamination area. Closeness relationship values were assigned based on the necessity of close proximity due to a logical flow of areas containing sequential operations. We applied this information to create the following relationship diagram. With the relationship diagram, we can now get a better feel for how to setup the facility.

The relationship diagram, reported in Fig. 5, was constructed based on the data derived from the activity relationship diagram. The "absolutely important" relationships were drawn first to ensure close proximity to one another, followed next by "especially important", "important" and "ok". With these relationships, we can now attempt to originate a facility layout for the decontamination area. 


\begin{tabular}{lll} 
Table 2: & Adjacency scoring and values \\
\hline \multicolumn{2}{l}{ Values } \\
\hline A & 64 & \\
E & 16 & \\
I & 4 \\
O & 1 & \\
U & 0 & \\
X & -1024 & \\
Adjacency Scoring & \\
A & $11 * 64=$ & 704 \\
E & $7 * 16=$ & 112 \\
I & $3 * 4=$ & 12 \\
O & $1 * 1=$ & 1 \\
U & $0 * 33=$ & 0 \\
X & $0 *-1024=$ & 0 \\
& Total $=$ & 829 \\
\hline
\end{tabular}

The above relationship diagram was determined through trial and error and reflects the best solution. Obviously other alternatives may be arranged. However, this is most desirable because it arranges the strongest relationships nearest to each other.

Adjacency scoring: The last score we need is the adjacency solution which will weigh the importance of each relationship considered in the previous two diagrams. The adjacency score calculated in Table 2 describes the weighted importance of each relationship. Using the weights for each letter relation, multiplied by the number of occurrences of each corresponding letter, the sum of these products yields the score. The desired outcome is maximization of the score. The scoring helps prevent the placement of undesirable areas near one another due to the weight of " $X$ " type relation which is a negative value. The score of 829 is the maximum result yielded from various diagrams.

Let's build the pre-er area: The following are the important points to consider when building the pre-ER area.

Lines: Having three lines to accommodate staff, males and females separately.

Advantages: The lines would accommodate more patient arrivals.

Disadvantages: There is a chance that all of the patients coming in would be of one gender. It is highly unlikely that three lines would be needed at once and

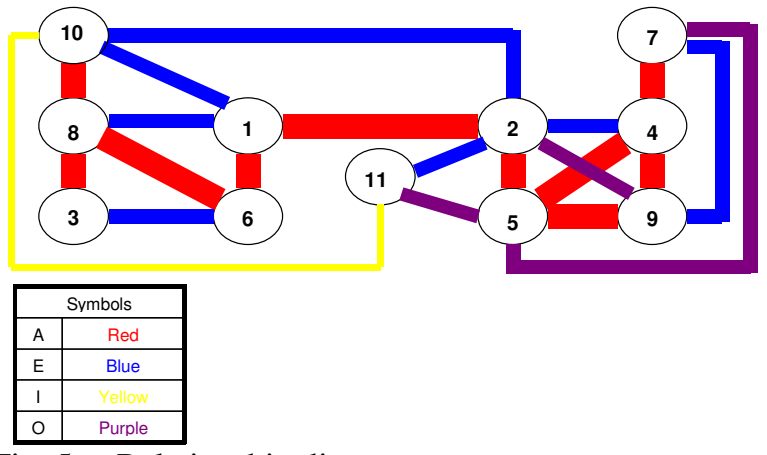

Fig. 5: Relationship diagram

therefore is an unnecessary waste of space. Having two general first in, first out (FIFO) lines.

Showers: Having combined showers between the lines.

Advantages: Only one containment and draining system would be necessary.

Disadvantages: Patients in different lines do not necessarily arrive at the same time. One shower could cause extensive queuing. Having a separate set of showers for each line.

Emergency track: Patients requiring the Emergency Track feature could be sent through the general decontamination process.

Advantages: Uninterrupted flow would result.

Disadvantages: Patients in critical conditions need immediate treatment. The amount of time each patient waits to be treated in the general decontamination line would increase due to an abundance of patients. Patients requiring the Emergency Track feature could be dropped off before the vehicle shower at contaminated stabilizing rooms.

Explanation of design selection: The selected layout gives a footprint of the emergency response area. Three lanes are provided for the ambulances to drive through.

Lane one, the outermost lane, accommodates the emergency track arrivals. This lane merges with Lane two (the middle lane) as it approaches the ambulance shower after dropping off its critical patients.

There is an additional lane, lane three, which is identical to lane 2 this provides for multiple arrivals. Lanes are wide enough to have room for ambulances as well as personal vehicles. Width of the lanes is 118 inches which accommodates the average ambulance size. 
Each station of decontamination is divided into five equal sections with hanging curtains as temporary partitions to maintain flexibility within the flow as well as patient privacy during decontamination. An idea of an ER plant is reported in Fig. 6 where the plan of a potential building realization is made. There are five showers to provide for two EMT's, an ambulance driver, a patient and an additional person or patient. The ambulance departs after being restocked as the decontaminated patients enter the emergency area. For unstable patients that need immediate care, they are immediately dropped off at a contaminated trauma room. Four beds occupy this trauma area; it is equivalent to two double occupancy resuscitation rooms. After patients are stabilized they are moved through the decontamination process with the same set up as non-critical patients who are then moved into a step down area where eight patients can be closely monitored. From each of the previous analyses, the determination of this alternative was the most logical and efficient.

Queuing analysis: This analysis was performed to check how long the patient will be in the emergency room (waiting time), how long it takes to see healthcare personnel (service time), the average patient waiting time to be "served" (waiting queue) and the average time waiting in line (time waiting in queue).

*Assuming 150 patients per day for 2 days with average service time of 14 minutes with two lines running.

$\lambda=75$ arrivals $/$ day $=0.0521$ arrivals $/ \mathrm{min}$

$\mu=2$ patients $/ 14 \mathrm{~min}=0.1429$ patients $/ \mathrm{min}$

Utilization factor

$\mathrm{p}=\lambda / \mu=0.365$ or $36.5 \%$

Probability of 0 units in system

Po $=1-\lambda / \mu=0.635$ or $63.5 \%$

Average number of patients in system (waiting and being served)

Ls $=\lambda /(\mu-\lambda)=0.574$ patients

Average time in system (waiting and service time)

$\mathrm{Ws}=1 /(\mu-\lambda)=11.02 \mathrm{~min}$.

Average number of patients waiting in queue

$\mathrm{Lq}=\lambda^{2} / \mu(\mu-\lambda)=0.209$ patients

Average time waiting in queue

$\mathrm{Wq}=\lambda / \mu(\mu-\lambda)=4.016 \mathrm{~min}$.

\section{DISCUSSION}

The most important aspect of implementation is recognizing that the hospital needs a response to external domestic or international disaster ${ }^{[10-11]}$. The emergency department is the key area that needs to be



Fig. 6: Flow diagram of how the ER should look

strengthened for this response. An emergency department needs a protected area to prevent secondary contamination of staff in the department ${ }^{[12]}$. Clear labeling of contaminated and clean linens is needed when an NBC response has been activated. All patients presenting after an NBC incident require decontamination. In the ambulance bay, there needs to be a cubicle with one showerhead. Further, three rows of two showerheads need to be installed to have more room for more people. For decontamination, patients are required initially to shower with their clothes on and then remove their clothing, step forward to the next showerhead and shower while gently cleansing with soap ${ }^{[13-14]}$. They then move forward to dry themselves and put on a clean disposable suit in the clean zone. The principle is to move at all times toward the clean zone. Staff puts the patient's belongings in a clean bag and the bag and the patient are labeled with identical numbers. The bags of belongings should then be placed in the contaminated clothing bin. All showers should have temperature regulation to prevent hypothermia and provide patient comfort. Having the showerhead in the ceiling of the ambulance bay allows decontamination to be carried out under cover. The ceiling should also be fitted with a sprinkler system that is isolated from the rest of the department so that it can be used if the shower proves inadequate. Once patients are decontaminated, they are allowed into the hospital. Depending on the triage category, they will go into the emergency department in a demarcated area ${ }^{[13-14]}$. 
It is very important to understand the concept that there is a reported risk from secondary exposure to contaminated patients at healthcare facilities. Even though this has been graded as a low risk, if there is not a good decontamination facility and there are many patients coming in at the same time to the same hospital, this risk can be very high. This risk should be adequately addressed with level III personnel protective equipment, including air purifier and respiratory technology, unless the facility determines that specific local threats require an increased level of protection ${ }^{[13-}$ 14].

Healthcare facilities are an essential component of an emergency response system but, at the present time, are poorly prepared for an incident. The greatest challenge may be the sudden presentation of a large number of contaminated individuals. Key elements of the healthcare facility response plan include prompt recognition of the incident, staff and facility protection, patient decontamination and triage, medical therapy and coordination with external emergency response and public health agencies. Controversial aspects include the optimal choice of personnel protective equipment, establishment of patient decontamination procedures, the role of chemical and biological agent detectors and potential environmental packs and water treatment systems. These all need to be done to improve our response strategies ${ }^{[11]}$.

The fundamental precept in hospital-based planning for bioterrorism events includes having a comprehensive emergency room disaster plan that is based on threat and vulnerability analysis. The JCAHO environmental healthcare standards and approach to disaster planning and management form the basis for a solid bioterrorism response plan. Preparation, education and training are imperative. Clinicians must maintain a high index of suspicion for use of bioterrorism agents, be able to make a rapid diagnosis and initiate empirical treatment ${ }^{[3-4]}$. However, the personnel, fellow administration, security, public relations, laboratory, pharmacy and facility management department should be familiar with the plan and know when to activate it. A recognized incident command system should be used. Hospital leadership must be aware of facility capabilities, capacity and should also have plans for expansion of service to meet the surge in demand. The command center should coordinate the emergency personnel teams, decontamination, security, acquisition of supplies and notification of public health and other authorities, including the media. If the plan is implemented, stress management and psychological support will play an important role in recovery ${ }^{[13-14]}$.

\section{CONCLUSION}

To our knowledge, this is the first attempt to have engineers look at an existing ER and set a mathematical model and plan to try to design the perfect ER and ER flow.

The medical profession must assume a central role and participate actively in the discussion and planning strategies, even with other specialists such as engineers, in order to influence formulation of policy that will activate in response to bioterrorism events.

From our model, we conclude to set up a new ER response we need:

* Having two general first in, first out (FIFO) lines

* Having a separate set of showers for each line

* Having a stable patient and unstable patient track will make it easy to respond to the emergency

* Patients requiring the emergency track feature would be dropped off before the vehicle shower at contaminated stabilizing rooms

Time of service: waiting 14 minutes with an average of 2 patients waiting in line to be served appears to be reasonable. Time and queue seems a reasonable starting and final point of the project. This is an initial study having a flow of 150 patients arriving over a long time period, but it is an attempt to a multidisciplinary approach to a clinical potential discussion.

\section{REFERENCES}

1. Brennan, R.J., J.F. Waeckerle, T.W. Sharp and S.R. Lillibridge, 1999. Chemical warfare agents: Emergency medical and emergency public health issues. Ann. Emerg. Med., 34: 191-204.

2. Bradley, R.N., 2000. Health care facility preparation for weapons of mass destruction. Prehosp. Emerg. Care., 4: 261-9.

3. Frezza, E.E., 2005. The challenge of the hospitals $\&$ healthcare systems in preparation for biological and chemical terrorism attack. J. Social Sci., 1: 1924.

4. Frezza, E.E., 2005. The quality of homeland security and healthcare systems: The Texas rangers initiative. J. Social Sci., 1: 194-196.

5. Macintyre, A.G., G.W. Christopher, E. Eitzen Jr., R. Gum, S. Weir, C. DeAtley, K. Tonat and J.A. Barbera, 2000. Weapons of mass destruction events with contaminated casualties: effective planning for health care facilities. JAMA, 283: 242-9. 
6. Schultz, C.H., J.L. Mothershead and M. Field, 2002. Bioterrorism preparedness. I: The emergency department and hospital. Emerg. Med. Clin. North Am., 20: 437-55.

7. McFee, R.B., 2002. Preparing for an era of weapons of mass destruction (WMD). Are we there yet? Why we should all be concerned. Part 1 . Vet. Hum. Toxicol., 44: 193-9.

8. Flowers, L.K., J.L. Mothershead and T.H. Blackwell, 2002. Bioterrorism preparedness. II: The community and emergency medical services systems. Emerg. Med. Clin. North Am., 20: 45776.

9. Brennan, R.J., J.F. Waeckerle, T.W. Sharp and S.R. Lillibridge, 1999. Chemical warfare agents: Emergency medical and emergency public health issues. Ann. Emerg. Med., 34: 191-204.

10. Bradley, R.N., 2000. Health care facility preparation for weapons of mass destruction. Prehosp. Emerg. Care, 4: 261-9.
11. Frezza, E.E., 2004. The challenge to hospitals and health care systems preparing for biological and chemical terrorism attacks. ACMQ Focus, 14: 1112.

12. Schultz, C.H., J.L. Mothershead and M. Field, 2002. Bioterrorism preparedness. I: The emergency department and hospital. Emerg. Med. Clin. North Am., 20: 437-55.

13. McFee, R.B., 2002. Preparing for an era of weapons of mass destruction (WMD). Are we there yet? Why we should all be concerned. Part 1 . Vet. Hum. Toxicol., 44: 193-9.

14. Flowers, L.K., J.L. Mothershead and T.H. Blackwell, 2002. Bioterrorism preparedness. II: The community and emergency medical services systems. Emerg. Med. Clin. North Am., 20: 45776. 